\title{
Селективный эпитаксиальный рост III-N-структур с использованием ионной нанолитографии
}

\author{
(C) В.В. Лундин ${ }^{1}$, А.Ф. Цацульников ${ }^{2}$, С.Н. Родин ${ }^{1}$, А.В. Сахаров ${ }^{1}$, С.О. Усов ${ }^{2}$, \\ М.И. Митрофранов ${ }^{1,2}$, Я.В. Левицкий ${ }^{1,2}$, В.П. Евтихиев ${ }^{1}$ \\ ${ }^{1}$ Физико-технический институт им. А.Ф. Иоффе Российской академии наук, \\ 194021 Санкт-Петербург, Россия \\ ${ }^{2}$ Научно-технологический центр микроэлектроники и субмикронных гетероструктур \\ Российской академии наук, \\ 194021 Санкт-Петербург, Россия \\ E-mail: Lundin@vpegroup.ioffe.ru
}

(Получена 12 марта 2018 г. Принята к печати 19 марта 2018 г.)

\begin{abstract}
Проведены исследования селективного эпитаксиального роста $\mathrm{GaN}$ методом газофазной эпитаксии из металлоорганических соединений с использованием ионно-лучевого травления. Исследованы частично маскированные эпитаксиальные слои $\mathrm{GaN}$, на поверхности которых в едином технологическом процессе осаждался тонкий слой $\mathrm{Si}_{3} \mathrm{~N}_{4}$, в котором ионным пучком формировались окна различной формы. Изучены режимы селективного эпитаксиального роста, и показано, что для формирования объектов субмикрометрового размера в условиях, когда суммарная площадь окон в маске мала относительно общей площади образца, длительность эпитаксии должна составлять 5-10с, что ухудшает воспроизводимость параметров эпитаксиального процесса. Также показано, что механизм селективного роста объектов, имеющих субмикрометровые размеры, значительно отличается от такового для планарных слоев и селективновыращенных слоев с размерами в единицы мкм и более. Исследовано влияние потоков прекурсоров (триметилгаллия и аммиака) на характер селективной эпитаксии. Исследованы возможности изменения топологии маски для реализации модельных объектов применительно к фотонным кристаллам, именно изучено влияние формы и ориентации окон в маске $\mathrm{Si}_{3} \mathrm{~N}_{4}$ на характер селективной эпитаксии.
\end{abstract}

DOI: 10.21883/FTP.2018.10.46467.8861

\section{1. Введение}

Селективная эпитаксия III-N-гетероструктур с субмикрометровыми размерами открывает широкие перспективы для создания различных устройств нанофотоники и наноэлектроники нового поколения на основе широкозонных соединений InAlGaN [1-6], в которых используется неоднородность физических свойств в трех измерениях, - например, квантовых фотонных логических устройств. Первым этапом в развитии этого направления является управляемое создание объектов с субмикрометровыми размерами в латеральном и вертикальном направлениях.

В общем случае процесс селективной эпитаксии состоит из следующих операций: формирование исходной подложки или планарного эпитаксиального слоя, полностью закрытого маскирующим материалом; формирование окон в маскирующем покрытии; селективный рост в сформированных окнах. Традиционно формирование окон в маскирующем покрытии производится методами фото- или электронной литографии, что требует нанесения и последующего удаления с поверхности соответственно фото- или электронных резистов. Их следы являются существенным загрязнением, которое может нарушить последующий процесс селективной эпитаксии.

В работе исследовался новый метод реализации технологии селективной субмикрометровой эпитаксии для соединений III-N, основанный на использовании сверхтонких маскирующих покрытий $\mathrm{Si}_{3} \mathrm{~N}_{4}$ толщиной 3-5 нм, осажденных в ректоре для газофазной эпитаксии из металлоорганических соединений (ГФЭМОС) непосредственно после завершения эпитаксиального роста $\mathrm{GaN}$ без прерывания технологического процесса. Ранее было установлено $[7,8]$, что формирующиеся слои $\mathrm{Si}_{3} \mathrm{~N}_{4}$ характеризуются хорошим пассивирующим эффектом, подавляя формирование электрически активных дефектов на поверхности III-N-структур, обладают планарностью на уровне моноатомного слоя и сплошностью при толщине $\sim 1$ нм.

На структурах $\mathrm{GaN} / \mathrm{Si}_{3} \mathrm{~N}_{4}$ с помощью локального травления сфокусированным ионным пучком осуществлялось формирование окон в маскирующем слое $\mathrm{Si}_{3} \mathrm{~N}_{4}$. Данный метод широко используется для формирования различных трехмерных структур субмикрометрового и нанометрового размеров $[9,10]$, но обработка больших площадей (порядка площади подложки диаметром 2-4дюйма) требует очень продолжительного времени, что осложняет использование данной технологии даже для прототипирования приборных структур. Однако положительность процесса стравливания тонкого слоя $\mathrm{Si}_{3} \mathrm{~N}_{4}$ в общем случае пропорциональна объему удаляемого материала, поэтому использование сверхтонких маскирующих слоев позволяет в десятки раз уменьшить необходимое для обработки подложки время. 


\section{2. Эксперимент}

В исследованных структурах сначала на подложке сапфира ориентации (0001) методом ГФЭМОС выращивался буферный слой $\mathrm{GaN}$ толщиной 3 мкм, легированный атомами кремния до концентрации $n=2 \cdot 10^{17} \mathrm{~cm}^{-3}$. После этого на поверхность слоя GaN in situ наносилась маска $\mathrm{Si}_{3} \mathrm{~N}_{4}$, при этом в качестве источников кремния и азота использовались силан $\left(\mathrm{SiH}_{4}\right)$ и аммиак $\left(\mathrm{NH}_{3}\right)$. Легирование буферного слоя необходимо для стока заряда при последующем ионном травлении, так как накапливающийся на поверхности электрический заряд может приводить к расфокусировке и отклонению ионного пучка. Окна в слое $\mathrm{Si}_{3} \mathrm{~N}_{4}$ формировались с помощью травления в сверхвысоком вакууме сфокусированным пучком ионов $\mathrm{Ga}$ с энергией 30 кэВ при токе 450 п $\mathrm{A}$, сфокусированным в пятно диаметром 40 нм [11], величина шероховатости травленой поверхности не превышала 2 нм. Доза облучения образца при травлении составляла 45 пКл/мкм² ${ }^{2}$ Сканирование пучком ионов Ga по поверхности позволяло формировать в маскирующем слое окна произвольной геометрии, различающиеся конфигурацией, взаимным расположением и геометрическими размерами. После формирования окон методом ГФЭМОС осуществлялся селективный рост GaN. B качестве источников азота и галлия использовались аммиак $\left(\mathrm{NH}_{3}\right)$ и триметилгаллий (ТМГ), а в качестве газа-носителя водород. Давление в реакторе при росте $\mathrm{GaN}$ составляло 100 мбар, температура подложки была $1030^{\circ} \mathrm{C}$.

\section{3. Результаты}

После формирования окон различной геометрии, проводилось селективное выращивание $\mathrm{GaN}$ методом ГФЭМОС в различных режимах. Целью исследований было определение влияния условий и продолжительности процесса селективной эпитаксии на характер данного процесса и свойства получаемых микро- и наноструктур $\mathrm{GaN}$. В используемом подходе с помощью локального ионно-лучевого травления через маску формируются отдельные элементы или весьма ограниченные массивы элементов, а при этом вся поверхность структуры между этими объектами остается закрытой маской. Однако если суммарная площадь окон в маске мала и осаждения $\mathrm{GaN}$ на маске не происходит, то приток материала к окнам оказывается очень большим за счет поверхностной диффузии по маске и малого обеднения газовой фазы. Это приводит к многократному превышению скорости роста $\mathrm{GaN}$ в окнах по сравнению со скоростью планарного роста в тех же условиях. В принципе подобные эффекты характерны для любой селективной эпитаксии, однако обычно отношение суммарной площади окон к полной поверхности составляет десятки процентов, а в нашем случае - сотые и тысячные доли процента. Таким образом, основной задачей исследований становилось изуче-
Режимы роста и площадь поперечного сечения полоска

\begin{tabular}{c|r|c|c|c}
\hline Образец & $\begin{array}{c}\text { Время } \\
\text { роста, с }\end{array}$ & $\begin{array}{c}\text { Поток ТМГ, } \\
\text { мкмоль/мин }\end{array}$ & $\begin{array}{c}\text { Поток } \mathrm{NH}_{3}, \\
\text { см}^{3} / \text { мин }\end{array}$ & $\begin{array}{c}\text { Площадь } \\
\text { поперечного } \\
\text { сечения, мкм }\end{array}$ \\
\hline 1 & 5 & 62.8 & 312 & $0.14-0.16$ \\
2 & 15 & 62.8 & 312 & $0.5-0.55$ \\
3 & 50 & 62.8 & 312 & $3.5-3.7$ \\
4 & 180 & 62.8 & 312 & $11.5-12.5$ \\
5 & 5 & 183.7 & 312 & $0.05-0.07$ \\
6 & 50 & 18.4 & 312 & $1.1-1.2$ \\
7 & 15 & 62.8 & 100 & $0.55-0.6$ \\
8 & 15 & 62.8 & 1000 & $0.48-0.52$
\end{tabular}

ние процесса формирования селективно-эпитаксиальных структур в условиях сильного переобогащения.

В первой серии экспериментов по эпитаксиальному росту изучалось развитие во времени селективновыращенных полосков, ориентированных вдоль направления $\langle 10 \overline{2} 0\rangle \mathrm{GaN}$, т.е. эпитаксиальные процессы проводились в идентичных условиях, но различались по продолжительности. Окна имели ширину $\sim 300$ нм и располагались в 20 мкм друг от друга. Массив полосков имел суммарный размер $4 \times 0.44$ мм. Вся остальная поверхность подложки была закрыта $\mathrm{Si}_{3} \mathrm{~N}_{4}$. В качестве режимов, исходных для проведения исследований, были выбраны ранее оптимизированные условия для обычной селективной эпитаксии $\mathrm{GaN}$ (см. таблицу, образец 1) [12,13]. В этих условиях скорость роста планарных слоев $\mathrm{GaN}$ составляет $\sim 5.5$ мкм/час. При обычной селективной эпитаксии данные условия приводят к однородному началу роста в окнах и малому боковому разрастанию.

Во всех практически используемых режимах ГФЭМОС GaN (за исключением осаждения низкотемпературных зародышевых слоев) рост $\mathrm{GaN}$ ограничен доставкой галлия к растущей поверхности (диффузионно-лимитированный рост). Это верно и для условий описываемых экспериментов. При этом скорость роста $\mathrm{GaN}$ пропорциональна концентрации ТМГ в атмосфере реактора за вычетом скорости травления (зависящей от температуры и концентраций водорода и аммиака). При селективной эпитаксии с относительно широкими окнами (единицы и десятки мкм) аналогично ведет себя скорость увеличения площади поперечного сечения полосков. Это означает, что площадь поперечного сечения полоска должна быть пропорциональна длительности осаждения, концентрации ТМГ и соответственно их произведению (экспозиции).

После проведения ряда предварительных экспериментов с различной длительностью эпитаксиального процесса, выявивших нелинейность зависимости площади поперечного сечения полосков от длительности эпитаксиального процесса, была проведена серия экспериментов с тщательным контролем идентичности условий выращивания, длительность которого составляла 5,15 , 
$a$

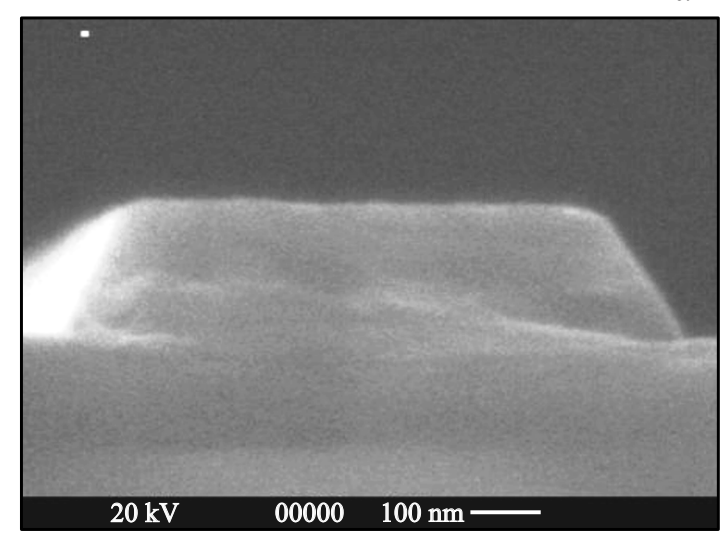

$c$

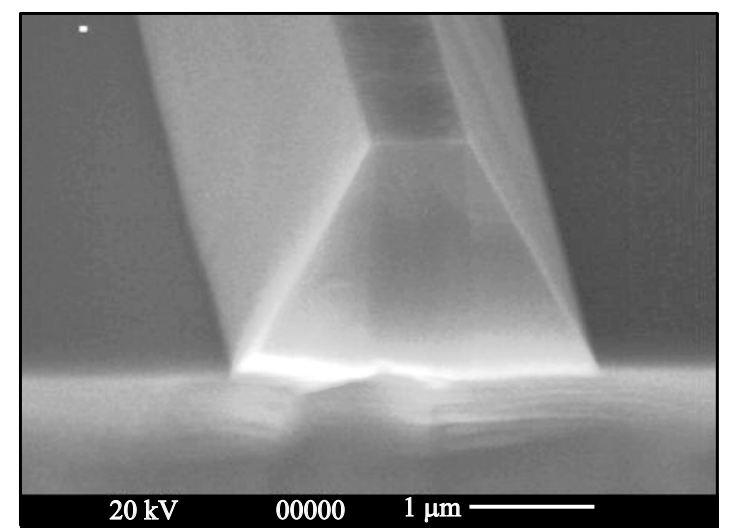

$b$

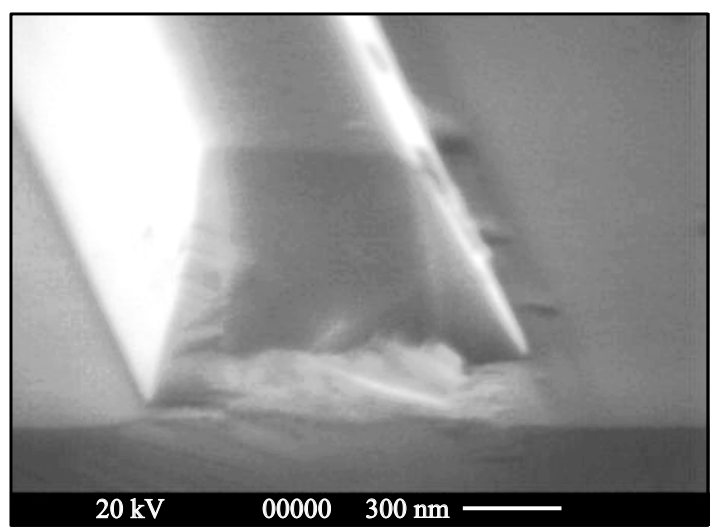

$d$

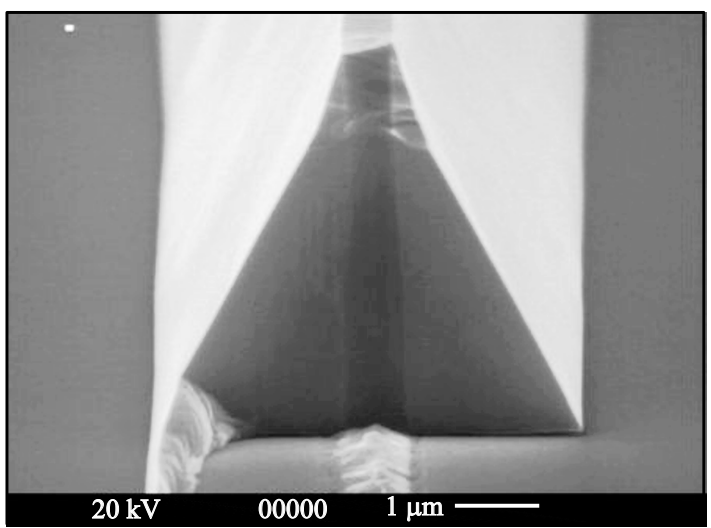

Рис. 1. РЭМ-фотографии селективно выращенных полосков $\mathrm{GaN}$ в окнах шириной $\sim 300$ нм. Время роста, с: $a-5, b-15, c-$ $50, d-180$.

50 и 180 с (образцы 1-4). Изображения таких полосков, полученные с помощью растровой электронной микроскопии (РЭМ), представлены на рис. 1.

Было установлено, что при малой длительности процесса селективной эпитаксии площадь поперечного сечения полоска $(S)$ возрастает во времени $(t)$ примерно как $S \propto t^{3 / 2}$ (рис. 2), а при большой длительности становится пропорциональной времени. Следует подчеркнуть, что при малой длительности эпитаксиального процесса количество встроившегося в полосок материала (площадь поперечного сечения) в 2-3 раза меньше ожидаемого для линейной зависимости, т. е. эффект отнюдь не мал и проявляет себя в самой актуальной области размеров - субмикрометровой. Кроме того, этот эффект воспроизводился в различных сериях экспериментов, что исключает ошибку одного-двух экспериментов, а результаты, приведенные на рис. 2, получены усреднением данных по многим полоскам.

Из полученных результатов можно сделать следующие выводы. Во-первых, для формирования объектов субмикрометрового размера в использованных режимах требуемая длительность эпитаксии должна составлять $5-10$ c, что находится в опасной близости к границе воспроизводимости. Во-вторых, механизм селективного роста таких объектов значительно отличается от такового для планарных слоев и селективно-выращенных объектов с размерами в единицы мкм и более.

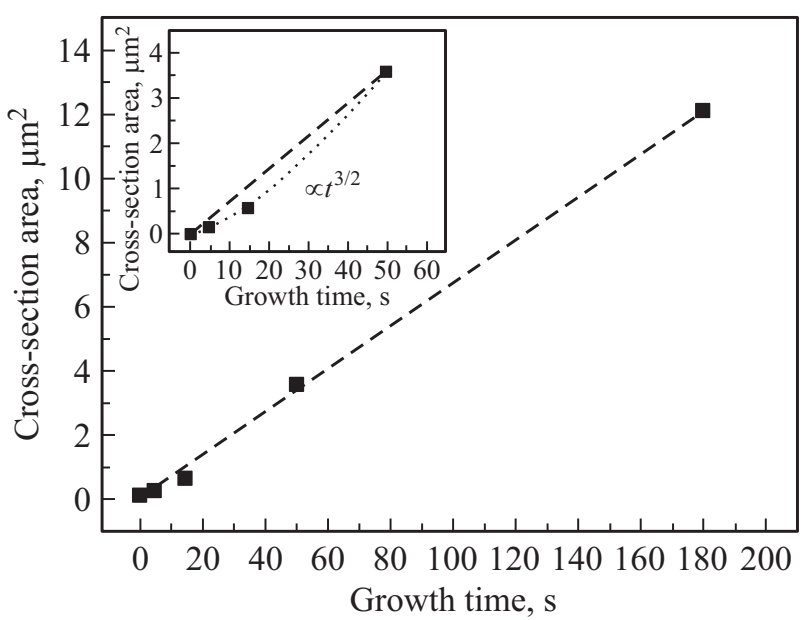

Рис. 2. Зависимость площади поперечного сечения полосков от длительности процесса селективной эпитаксии. На вставке: точки - аппроксимация экспериментальных результатов зависимостью $S \propto t^{3 / 2}$. 
$a$

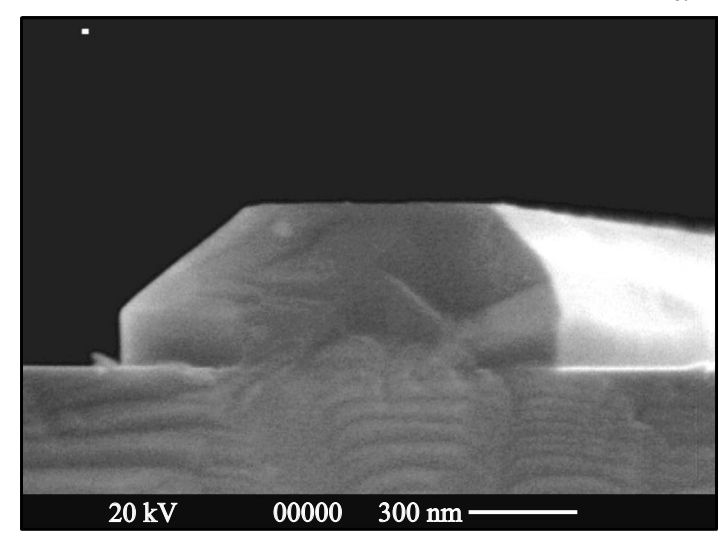

$b$

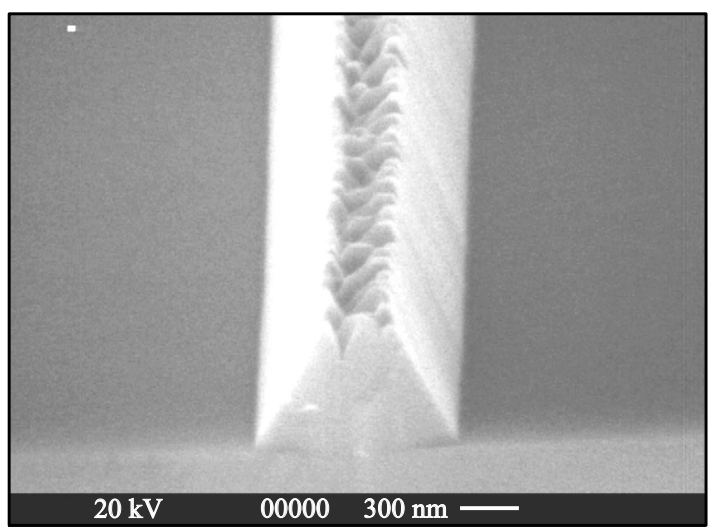

Рис. 3. РЭМ-фотографии селективно выращенных полосков GaN. Время роста 15 с. a- образец 7 (малый поток $\mathrm{NH}_{3}$ ), $b$ - образец 8 (большой поток $\mathrm{NH}_{3}$ ).

Для получения дополнительной информации о процессе селективного роста и в поисках путей увеличения длительности эпитаксиального процесса при сохранении субмикрометровых размеров было изучено влияние потоков прекурсоров (ТМГ и $\mathrm{NH}_{3}$ ) на характер селективной эпитаксии. Образец 1 это вышеописанная структура с длительностью эпитаксиального процесса 15 c. При выращивании образцов 5 и 6 потоки ТМГ и длительность эпитаксиального процесса изменялись в 3 раза таким образом, чтобы произведение этих величин (экспозиция) оставалось постоянным. Полученная площадь поперечного сечения полоска приведена в таблице. Видно, что при неизменной экспозиции увеличение потока ТМГ приводит к увеличению количества осажденного материала (площади поперечного сечения полоска). Следует отметить, что при наименьшем потоке ТМГ нарушается сплошность полосков - наблюдается фрагментарный рост. В этом случае за площадь поперечного сечения, приведенную в таблице, принята средняя высота полоска там, где он есть. Площадь поперечного сечения полосков незначительно снижается при увеличении потока аммиака, что связано с уменьшением коэффициента диффузии галлийсодержащих компонент в газовой фазе при увеличении концентрации аммиака в водороде (аналогичная зависимость наблюдается и при планарной эпитаксии). В то же время поток (концентрация) аммиака существенно влияет на его профиль. При сравнении РЭМ-изображений полосков, выращенных при различных потоках аммиака (рис. 1, $b$, $3, a$ и $b$ ), видно, что при малом потоке $\mathrm{NH}_{3}$ у полосков появляются дополнительные боковые вертикальные грани (их высота равна примерно половине всей высоты полоска) и увеличивается ширина полоска при уменьшении его высоты (рис. 3, a). При большом потоке аммиака не происходило планаризации верхней грани полоска (рис. $3, b)$.

Рассмотрим возможные причины, объясняющие наблюдаемые эффекты.

Во-первых, транспорт галлийсодержащих молекул в газовой фазе к поверхности малой площади затруд- нен („диффузия в нулевой радиус“). Это может объяснить сверхлинейный характер зависимости поперечного сечения полоска от длительности эпитаксиального процесса при их малых размерах (малых длительностях). Однако скорость набора материала все равно должна быть пропорциональна концентрации ТМГ в реакторе, что противоречит прямым экспериментам.

Во-вторых, малость площади поверхности полоска в начале роста в сочетании с крайне высоким переобогащением газовой фазы может приводить к тому, что галлий не успевает выстроиться или поверхность пассивируется продуктами реакции (рост ограничивается скоростью реакции синтеза $\mathrm{GaN}$ или ухода продуктов реакции). Однако в этом случае увеличение концентрации ТМГ в реакторе не должно приводить к увеличению скорости роста полосков, что также противоречит прямым экспериментам.

Таким образом, мы вынуждены сделать заключение, что встраивание галлия в $\mathrm{GaN}$ при крайне малых размерах полосков ограничено каким-то еще не изученным механизмом. Возможно, оно связано с преодолением потенциального барьера. При средних концентрациях ТМГ это снижает скорость роста, а при малых концентрациях ТМГ подавляет устойчивый рост. Детальное изучение данного эффекта планируется в последующих работах. Конечной практической целью является нахождение режимов роста, позволяющих воспроизводимо формировать субмикрометровые эпитаксиальные структуры при длительности процесса $>30 \mathrm{c}$.

Во второй серии экспериментов в данной работе изучалось влияние формы и ориентации окон, вытравленных в $\mathrm{Si}_{3} \mathrm{~N}_{4}$, на характер селективной эпитаксии. Данные исследования необходимы для определения возможности создания „библиотеки“ базовых элементов квантовых фотонных логических устройств. Были изучены следующие группы объектов:

— „веер полосков“ (рис. 4,a) - 8 полосковых окон длиной 1 мм и шириной $\sim 150$ нм, выходящих из одной 
$a$

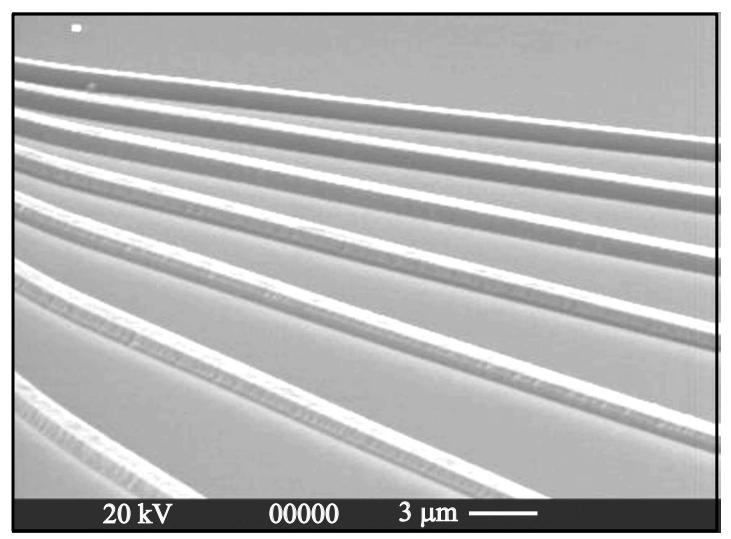

$c$

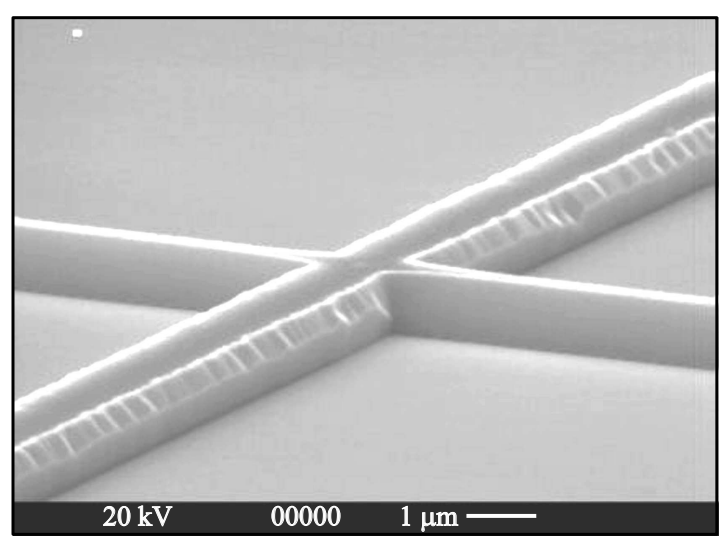

$b$
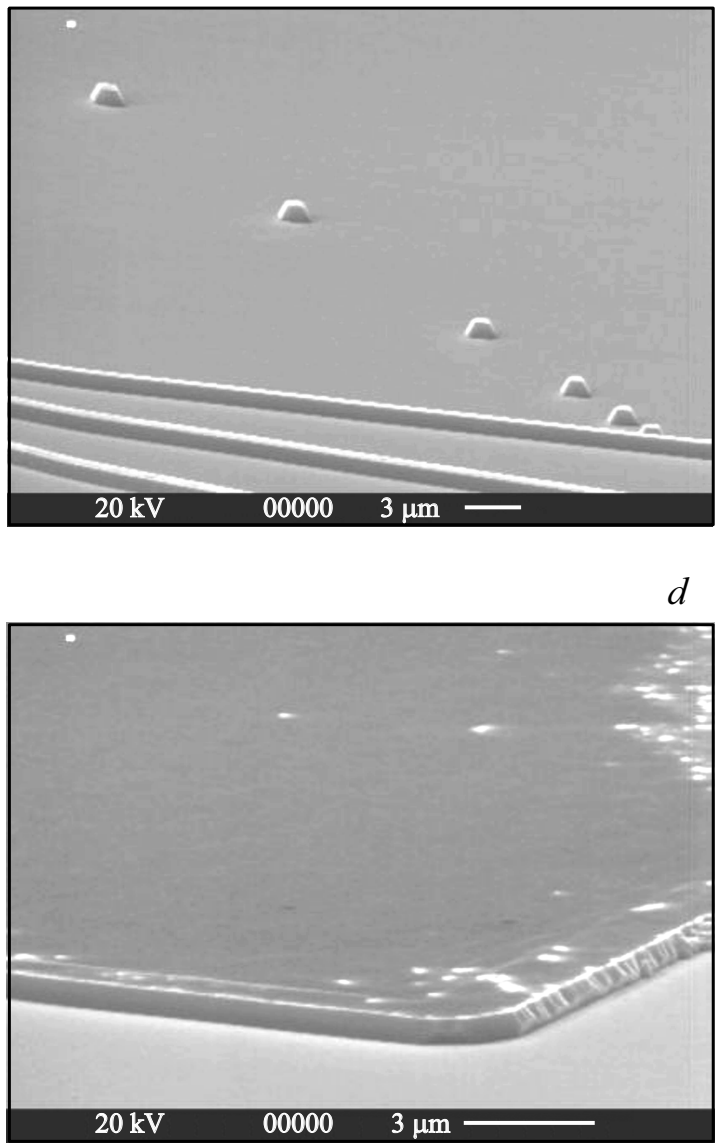

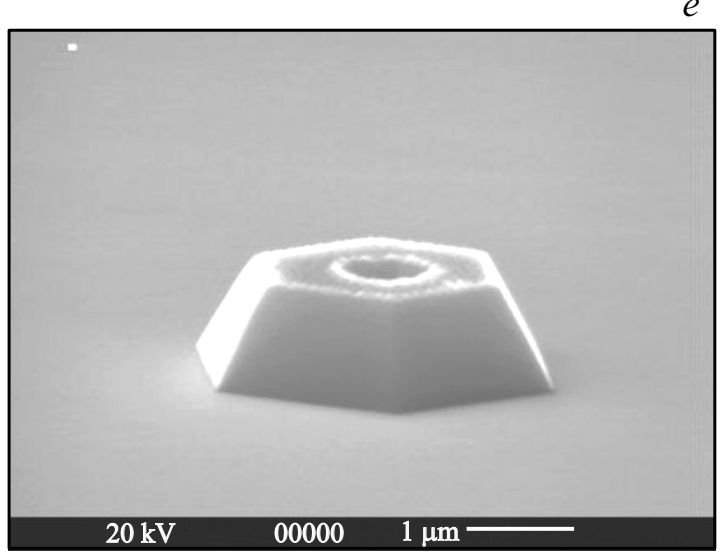

Рис. 4. GaN-структуры, выращенные в окнах различной формы. $a-$ „веер полосков“, $b-4$ группы разноудаленных от веера полосков круглых окон, $c-$ „кресты“, $d-$ квадратные окна, $e-$ круглые и кольцевые окна. Время роста 15 с.

точки (первый полосок ориентирован вдоль направления $\langle 11 \overline{2} 0\rangle \mathrm{GaN}$ и угол между соседними полосками составляет $7.5^{\circ}$, т. е. последний полосок тоже ориентирован вдоль $\langle 11 \overline{2} 0\rangle \mathrm{GaN})$;

- 4 группы разноудаленных от веера полосков круглых окон диаметром 500 нм (рис. 4, $b$ ) (отверстия в каждой группе последовательно удалены от первого полоска на 2, 5, 10, 20, 40, 60 мкм; группы располагаются через одинаковые промежутки, первая - у основания веера, последняя начинается от конца полоска);

- взаимно перпендикулярные полосковые окна („кресты“, рис. 4,c, один из полосков ориентирован вдоль $\langle 11 \overline{2} 0\rangle \mathrm{GaN})$;

- сплошные квадратные макроскопические окна (рис. 4,d) размером $200 \times 200$ мкм, ориентированные одной стороной вдоль $\langle 11 \overline{2} 0\rangle \mathrm{GaN}$;

- круглые и кольцевые окна (рис. $4, e)$. 
Режимы эпитаксиального роста для всех вариантов окон соответствовали образцам 1 и 4 таблицы (длительность роста 15 и $180 \mathrm{c}$ ).

Эксперименты с первой группой окон („веер“, рис. $4, a)$ позволили изучить влияние ориентации полоска, а также ширины маскированной области между полосками на характер эпитаксиального роста. Установлено, что полоски, ориентированные вдоль направления $\langle 11 \overline{2} 0\rangle \mathrm{GaN}$, в поперечном сечении представляют собой трапецию. При отклонении от этого направления у полосков возникают дополнительные вертикальные боковые грани. Наибольшей величины (до половины всей высоты) эти грани достигают у полоска, ориентированного вдоль направления $\langle 10 \overline{1} 0\rangle \mathrm{GaN}\left(30^{\circ}\right.$ к $\langle 11 \overline{2} 0\rangle)$. Наибольшая ширина наблюдается у полосков, ориентированных под углом $7.5-22.5^{\circ}$ к указанным низкоиндексным направлениям, что вполне естественно - в этом случае резко увеличивается плотность моноатомных ступеней на боковых стенках. Аналогичные результаты мы наблюдали и ранее при изучении селективной эпитаксии в окнах шириной в единицы мкм. Очень важным результатом является почти полное постоянство ширины и высоты полосков по их длине при малой продолжительности эпитаксиального процесса $(15$ c), т. е. ширина маскированной области не влияет на процесс роста, снова указывая на существование некоего лимитирующего механизма. При большой длительности эпитаксиального роста $(180 \mathrm{c})$ полоски уширяются по мере удаления от центра веера, что указывает на классический характер селективного роста: чем больше расстояние между полосками, тем с большей площади собирается галлий, тем больше ширина полоска.

Аналогичное поведение наблюдалось для второй группы окон (разноудаленные от полоска круглые окна): при малой продолжительности роста все кристаллиты, сформированные в окнах, имеют приблизительно одинаковые размеры (рис. $4, b)$, а при большой продолжительности роста, чем дальше кристаллит отстоит от полоска и других кристаллитов, тем он больше. Рост в крестообразных масках (рис. 4,c) показывает, что в пересечении полосков не возникает каких-либо дополнительных особенностей, что открывает путь к формированию сложных рисунков методом селективной эпитаксии. Рост в макроскопическом окне носит нормальный характер, показывая, что такое применение разрабатываемой технологии тоже возможно (рис. 4, $d$ ). В „чистом виде“ оно неэффективно (намного проще сформировать такие окна обычной фотолитографией и реактивно-ионным травлением), но может быть использовано в сочетании с субмикрометровыми окнами, сформированными в том же процессе. Рост в круглых и кольцевых окнах показал возможность формирования соответствующих кристаллитов $\mathrm{GaN}$ (рис. 4,e) и их матриц. На данном этапе развития технологии из-за избыточной скорости роста они получаются относительно крупными. В дальнейшем мы планируем найти возможность понижения скорости роста, что позволит контролировать их размеры.

\section{4. Заключение}

Таким образом, разработаны технологические методы селективной эпитаксии, основанные на комбинации осаждения in situ тонких маскирующих покрытий $\mathrm{Si}_{3} \mathrm{~N}_{4}$ на эпитаксиальных слоях $\mathrm{GaN}$, ионно-лучевого травления для формирования окон в маске и селективной эпитаксии в этих окнах. Было показано, что осажденный in situ $\mathrm{Si}_{3} \mathrm{~N}_{4}$ позволяет добиться сплошности маскирующего слоя при его сверхмалой толщине и только сверхмалая толщина маскирующего покрытия позволяет эффективно использовать ионно-лучевое травление для формирования окон. Показана возможность формирования различных субмикрометровых объектов $\mathrm{GaN}$ с помощью данного сочетания технологических приемов. В свою очередь это позволило перейти к субмикрометровым размерам селективно выращиваемых объектов и обнаружить, что в данном диапазоне размеров процесс роста существенно отличается от роста более крупных объектов.

Работа выполнена при поддержке проекта РФФИ № 17-02-01099-a.

\section{Список литературы}

[1] K. Wu, T. Wei, D. Lan, X. Wei, H. Zheng, Y. Chen, H. Lu, K. Huang, J. Wang, Y. Luo, J. Li. Appl. Phys. Lett., 103, 241107 (2013).

[2] L. Zhao, T. Wei, J. Wang, Q. Yan, Y. Zeng, J. Li. J. Semiconductors, 34, 104005 (2013).

[3] F. Qian, S. Gradecak, Y. Li, C.-Y. Wen, C.M. Lieber. Nano Lett., 5, 2287 (2005).

[4] A. Motayed, A.V. Davydov, M. He, S.N. Mohammad, J. Melngailis. Appl. Phys. Lett., 90, 183120 (2007).

[5] M. Abul Khayera, Roger K. Lake. J. Appl. Phys., 108, 104503 (2010).

[6] C.Y. Chen, G. Zhu, Y. Hu, J.-W. Yu, J. Song, K.-Y. Cheng, L.-H. Peng, L.J. Chou, Z.L. Wang. ACS Nano, 6, 5687 (2012).

[7] J. Derluyn, S. Boeykens, K. Cheng, R. Vandersmissen, J. Das, W. Ruythooren, S. Degroote, M.R. Leys, M. Germain, G. Borghs. J. Appl. Phys., 98, 054501 (2005).

[8] W.V. Lundin, S.N. Rodin, E.E. Zavarin, A.V. Sakharov, M.A. Sinitsyn, A.F. Tsatsulnikov. Proc. EW-MOVPE 2017 (Grenoble, 2017).

[9] J. Gierak. Semicond. Sci. Technol., 24 (4), 043001 (2009).

[10] P. Kitslaara, M. Strassner, I. Sagnes, E. Bourhis, X. Lafosse, C. Ulysse, C. David, R. Jede, L. Bruchhaus, J. Gierak. Microelectronic Engin., 83 (4-9), 811 (2006).

[11] M.I. Mitrofanov, S.N. Rodin, I.V. Levitskii, S.I. Troshkov, A.V. Sakharov, W.V. Lundin, V.P. Evtikhiev. IOP Conf. Series: J. Phys. Conf. Ser., 816, 012009 (2017).

[12] М.М. Рожавская, В.В. Лундин, А.В. Сахаров. Письма ЖТФ, 40 (1), 37 (2014).

[13] M.M. Rozhavskaya, W.V. Lundin, A.E. Nikolaev, E.E. Zavarin, S.I. Troshkov, P.N. Brunkov, A.F. Tsatsulnikov. Phis. Status Solidi C, 10 (3), 373 (2013).

Редактор Л.В. Шаронова 


\section{Selective epitxial growth of III-N structures using ion beam nanolithography}

W.V. Lundin ${ }^{1}$, A.F. Tsatsulnikov ${ }^{2}$, S.N. Rodin ${ }^{1}$, A.V. Sakharov' ${ }^{1}$, S.O. Usov' ${ }^{2}$, M.I. Mitrofanov ${ }^{1,2}$, I.V. Levitskii ${ }^{1,2}$, V.P. Evtikhiev ${ }^{1}$

${ }^{1}$ loffe Institute, 194021 St. Petersburg, Russia

${ }^{2}$ Submicron Heterostructures for Microelectronics, Research \& Engineering Center, Russian Academy of Sciences, 194021 St. Petersburg, Russia

\footnotetext{
Abstract Selective epitaxial growth of GaN by MOCVD technique with using ion beam etching was investigated. It were studied partially masked $\mathrm{GaN}$ epitaxial layers containing on the surface windows with different forms which were formed by ion beam in $\mathrm{Si}_{3} \mathrm{~N}_{4}$ layer deposited on the GaN layer in the single epitaxial process. Regimes of the selective epitaxial growth were studied, and it was shown that for formation of submicrometer objects under conditions when the total area of the windows in the mask is small relative to the total area of the sample, the required duration of the epitaxy should be $5-10 \mathrm{~s}$, that worsens the reproducibility of the parameters of the epitaxial process. It was also shown that the mechanism of the selective growth of the submicrometer objects is significantly different from that for planar layers and selectively grown layers with dimensions of $\sim 1 \mu \mathrm{m}$ or more. The effect of precursor fluxes (trimethylgallium and ammonia) on the selective epitaxy was studied. The effect of mask topology (influence of the shape and orientation of windows in the $\mathrm{Si}_{3} \mathrm{~N}_{4}$ mask on the character of selective epitaxy) on the realization of model objects for photonic crystals was studied.
} 